DEPÓSITO LEGAL ZU2020000153

Esta publicación científica en formato digital

es continuidad de la revista impresa

ISSN 0041-8811

E-ISSN 2665-0428

Revista

de la

Universidad

del Tunlia

Fundada en 1947

por el Dr. Jesús Emrique Lossada

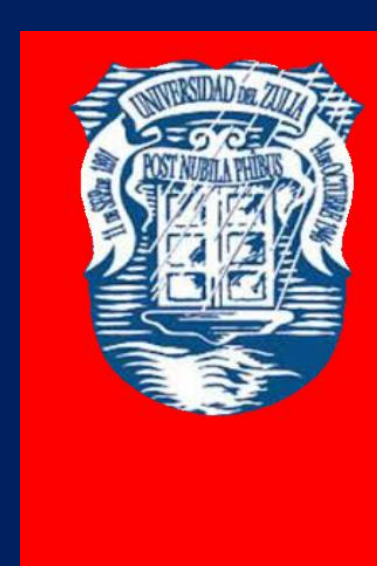

Ciencias

Sociales

y Arte

Aกัต 11 No 31

Septiembre - Diciembre 2021

Tercera ípoca

Maracailbo-Venezuela 
REVISTA DE LA UNIVERSIDAD DEL ZULIA. 3ª época. Año 11 N 31, 2020

Jhony Ostos \& Antonieta Hamann /// Responsabilidad social empresarial ...160-181

DOI: http://dx.doi.org/10.46925//rdluz.31.12

\title{
Responsabilidad social empresarial y el desempeño financiero en las mejores empresas para trabajar de un mercado emergente
}

\author{
Jhony Ostos * \\ Antonieta Hamann **
}

RESUMEN

Los programas de responsabilidad social de las empresas cada vez son más valorados por los clientes, sin embargo, su justificación financiera en las organizaciones aún sigue siendo limitada. El diseño de esta investigación fue basado en un estudio cualitativo de casos comparativos entre las mejores empresas para trabajar del Perú (Great place to work - GPTW) en el período 20152017. Se examinaron las prácticas de responsabilidad social y los resultados financieros obtenidos de cada una. Los resultados indican que existe una tendencia en las empresas que ejecutan programas de responsabilidad social, a obtener a la vez resultados financieros positivos.

PALABRAS CLAVE: Desempeño financiero; Great Place to Work; norma ISO 26000; responsabilidad social empresarial; responsabilidad social interna.

\section{Corporate social responsibility and financial performance in the best companies to work in an emerging market}

ABSTRACT

Corporate social responsibility programs are increasingly valued by customers, however their financial justification in organizations is still limited. The design of this research was based on a qualitative study of comparative cases between the best companies to work in Peru (Great place to work - GPTW) in the period 2015-2017. The social responsibility practices and the financial results obtained from each were examined. The results indicate that there is a tendency for companies that execute social responsibility programs to obtain positive financial results at the same time.

KEYWORDS: Corporate social responsibility; Great Place to Work; ISO 26000; financial performance; internal social responsibility.

* Ph.D. in Management Sciences. Profesor de la Facultad de Ciencias Económicas y Administrativas, Universidad Esan, Lima, Perú. ORCID: http://0000-0003-1888-7378. E-mail: jostos@esan.edu.pe

** Doctora en Turismo. Profesora de la Facultad de Ciencias Económicas y Administrativas, Universidad Esan, Lima, Perú ORCID: http://000-0001-9942-6877. E-mail: ahamann@esan.edu.pe

Recibido: 22/07/2020

Aceptado: 02/09/2020 
REVISTA DE LA UNIVERSIDAD DEL ZULIA. 3a época. Año $11 \mathrm{~N}^{\circ}$ 31, 2020 Jhony Ostos \& Antonieta Hamann /// Responsabilidad social empresarial ...160-181

DOI: http://dx.doi.org/10.46925//rdluz.31.12

Introducción

La responsabilidad social se ha convertido en un efecto adoptado por más y más empresas de todas partes del mundo como respuesta directa a desafíos que repercuten dentro y fuera de la comunidad empresarial, tales como el cambio climático, la cohesión social y la pobreza global. Por un lado, las prácticas de responsabilidad social empresarial (RSE) actual son un indicador de la preocupación de los gerentes por atender las expectativas sociales, y de otro lado, los consumidores cada vez más están conscientes y valoran las prácticas de RSE. Actualmente, el problema social se ha convertido en una preocupación importante en la actividad económica de las organizaciones, el cual se debe a que la organización moderna es evaluada también desde el punto de vista de contribución a la vida social de la comunidad (Muscalu et al, 2012; Martínez de Carrasquero, 2019).

El Instituto Internacional para el Desarrollo Sostenible ha identificado posibles beneficios clave de las empresas que implementan estrategias de RRSS: menores riesgos, reputación, manejo de recursos humanos, innovación, competitividad y posición en el mercado, eficiencia operativa, eficiencia en la cadena de suministros, posición para el cambio, mejor "licencia social", acceso al capital; relaciones legales, y promoción del consumo responsable (Hohnen, 2007). Además, explica que las razones para que una empresa dedique tiempo y recursos en prácticas de RSE son diversas, pudiendo ser: altruismo, interés personal, espíritu comercial, competencia y resultados financieros.

Según el informe del barómetro de confianza de Edelman (2017), el 81 \% de los miembros de la sociedad están preocupados y temerosos por los valores y problemas sociales. Numerosos autores e instituciones sostienen que las prácticas de responsabilidad social de las empresas aseguran una opinión pública favorable de los consumidores y de la sociedad, el cual se traduce luego en resultados financieros positivos tanto de corto y largo plazo; de otro lado, otros autores indican que las prácticas de responsabilidad social de las empresas, en muchos casos, pertenecen a actividades que no corresponden a su principal propuesta de valor, motivo por el cual, tienden a desanimarse y ponen en tela de juicio esta actividad; motivo por el cual Woodroof et al (2019) sostienen que el impacto de la RSE en el resultado financiero de las empresas aún es limitada y requiere continuar con su indagación. 
REVISTA DE LA UNIVERSIDAD DEL ZULIA. 3ª época. Año ll N 31, 2020 Jhony Ostos \& Antonieta Hamann /// Responsabilidad social empresarial ...160-181

DOI: http://dx.doi.org/10.46925//rdluz.31.12

En este contexto, este estudio tiene como objetivo examinar las prácticas de responsabilidad social empresarial y los resultados financieros obtenidos de una muestra de las mejores empresas para trabajar en el Perú. La muestra fue obtenida del reporte del instituto Great Place to Work, en el período 2015 - 2017. La guía para observar las acciones de responsabilidad social de las empresas fue el cumplimiento de las siete materias principales indicadas en la norma ISO 26000, el cual sirvió de guía para evaluar las acciones de responsabilidad social de las organizaciones de la muestra de estudio en el período 2015 - 2017, luego se hizo un análisis de los resultados financieros obtenidos en el mismo período de estudio.

Esta investigación es significante por lo siguiente: A) este estudio explora las actividades de responsabilidad social empresarial de las mejores empresas para trabajar de un mercado emergente, analizando si cumplen con los lineamientos de la norma ISO 26000. B) se evalúan los resultados financieros de las mejores empresas para trabajar de un mercado emergente y se examina si hay reciprocidad con las actividades de RSE.

\section{Revisión de la literatura}

El enfoque de una política de RSE dentro de una empresa puede basarse en factores internos, tales como la cultura organizacional, los recursos humanos, las estrategias, y otros; y en factores externos, tales como el cuidado del medio ambiente por: el cambio climático, la contaminación, oposición a la globalización, la crisis energética, los consumidores, las autoridades públicas, las ONG, los sindicatos, competidores, proveedores, y otros (Muscalu et al., 2012).

\subsection{Responsabilidad social empresarial (RSE)}

Los objetivos corporativos orientados al bienestar atraen cada vez más la atención del interés público, esto lleva a las empresas a tener un nuevo equilibrio de objetivos económicos, ambientales, y sociales; el compromiso voluntario de los objetivos no económicos que van más allá de los requisitos legales se denomina responsabilidad social empresarial (Schmitz y Schrader, 2015). Un problema para las empresas que adoptan políticas de RSE, es encontrar un equilibrio entre las prácticas de RSE y las actividades competitivas para mantener una posición sólida en entornos empresariales (Valiorgue et al, 2017). En este sentido, Barić (2017) sostiene 
REVISTA DE LA UNIVERSIDAD DEL ZULIA. 3ª época. Año ll N 31, 2020 Jhony Ostos \& Antonieta Hamann /// Responsabilidad social empresarial ...160-181

DOI: $\underline{\text { http://dx.doi.org/10.46925//rdluz.31.12 }}$

que muchas compañías utilizan las actividades de responsabilidad social como estrategia de diferenciación para obtener ventajas competitivas sostenibles y generar beneficios. De otro lado, Scandelius y Cohen (2016) indican que las empresas orientadas a la responsabilidad social están adoptando un enfoque estratégico más holístico, tomando en cuenta una cadena de valor más amplia y el ciclo de vida de los productos.

Rezaee (2017) sostiene que las actividades financieras y no financieras de sostenibilidad y responsabilidad social que realizan las empresas son complementarias, esto debido a que la empresa correctamente administrada se adhiere a sus principios éticos y ejecuta la RSE para generar resultados financieros a largo plazo.

\subsection{Responsabilidad social empresarial externa (RSEE)}

La responsabilidad social empresarial (RSE) es un término que describe las acciones de las organizaciones con respecto a las preocupaciones sociales y ambientales, y cómo estas acciones están relacionadas con las partes interesadas de cada organización (empleados, clientes, gobierno y actores sociales secundarios); una organización que es responsable de sus acciones y busca cooperar en el bienestar de su comunidad y el medio ambiente es una organización en la que se puede confiar (Su et al, 2019). Godos et al (2018) sugieren que las empresas deberían crear un comité específico que tenga a su cargo los asuntos sociales y ambientales, quienes deberían encargarse de supervisar las políticas y prácticas de RSE de los gerentes, para alentar la buena toma de decisiones sociales y ambientales que aseguren los intereses a largo plazo de la empresa, al respecto señalan que existen fuentes de asesoramiento externo que promueven en los miembros de la junta un mayor acercamiento de las partes interesadas de las empresas.

Park et al (2014) indican que una mala reputación provocada por la comunidad local es fatal para las filiales de empresas multinacionales (EMN) que sufren las responsabilidades sociales de sus empresas en el extranjero y, por lo tanto, puede ser un desencadenante que induce al fracaso de la inversión, por ello, la comunidad local juega un papel esencial en las actividades de RSE de las EMN, al igual que las organizaciones no gubernamentales (ONG) que pueden impulsar a las EMN a operar las filiales de manera socialmente responsable en los mercados locales. Los gobiernos tienen una influencia esencial directa e indirecta en la toma de decisiones 
REVISTA DE LA UNIVERSIDAD DEL ZULIA. 3ª época. Año ll N 31, 2020 Jhony Ostos \& Antonieta Hamann /// Responsabilidad social empresarial ...160-181

DOI: http://dx.doi.org/10.46925//rdluz.31.12

y el comportamiento social de las empresas a través de la configuración del marco legal y regulatorio; de otro lado, las partes interesadas también tienen influencia en la toma de decisiones de los gerentes, el cual puede conducir a tener una sólida reputación empresarial y por ende mejorar el rendimiento del negocio (Taghian et al, 2015). Los factores externos pueden afectar las decisiones de RSE de la organización, por lo tanto, se deben tener en cuenta estos factores para adoptar un enfoque particular de RSE, es decir se debe combinar la perspectiva de las partes interesadas y la perspectiva de la empresa, encontrándose tres factores a seguir explorando: gobierno, sociedad y negocios Vashchenko (2017).

Scandelius y Cohen (2016) sostienen que a veces para avanzar hacia el desarrollo empresarial sostenible, se requieren acciones empresariales en forma de iniciativas, innovación y colaboración/co-creación para mejorar los niveles de confianza entre la empresa y las partes interesadas. Park et al. (2014) examinaron en Corea del Sur, la relación de las partes interesadas con las prácticas de responsabilidad social empresarial (RSE) de las empresas multinacionales (EMN), encontraron una relación positiva entre las partes interesadas (gobierno, medios de comunicación, comunidad local y ONG) y las prácticas de RSE.

Taghian et al (2015) sugieren que las partes interesadas internas (empleados, sindicatos) y las partes interesadas externas (sociedad, medios de comunicación, y gobierno) influyen en la reputación empresarial. Godos et al. (2018) indican que existen elementos que influyen positivamente en el desarrollo e implementación de prácticas de RSE, dentro de ellas se tiene, a) la existencia de un comité específico de responsabilidad social y ambiental, b) el establecimiento de un marco legal de actuación, y c) la asesoría externa.

\subsection{Responsabilidad social empresarial interna (RSEI)}

Jaramillo (2011), citado en López et al (2014), indica que la primera responsabilidad social de la empresa debería ser con sus trabajadores, es decir se deberían respetar y cumplir: los derechos de los trabajadores, la legislación laboral, las normas de la organización internacional del trabajo (OIT), el desarrollo personal y profesional de los empleados, las condiciones y calidad de vida en el trabajo, y manifestando un compromiso con el respeto a los derechos humanos y laborales. En el cuadro 1 se resumen los conceptos de RSEI brindado por diferentes autores. 
REVISTA DE LA UNIVERSIDAD DEL ZULIA. 3ª época. Año 11 N 31, 2020 Jhony Ostos \& Antonieta Hamann /// Responsabilidad social empresarial ...160-181

DOI: http://dx.doi.org/10.46925//rdluz.31.12

Cuadro 1: Responsabilidad social empresarial interna (RSEI)

\begin{tabular}{|l|l|}
\hline \multicolumn{1}{|c|}{ Autores } & \multicolumn{1}{|c|}{ Descripción } \\
\hline Bestratén \& Pujol (2004) & $\begin{array}{l}\text { Se refiere a las responsabilidades contraídas con los } \\
\text { trabajadores y con la comunidad. Se manifiestan como } \\
\text { responsabilidades secundarias que en algunos casos } \\
\text { exceden lo obligatorio }\end{array}$ \\
\hline $\begin{array}{l}\text { Libro verde la Unión Europea } \\
(2002)\end{array}$ & $\begin{array}{l}\text { La responsabilidad interna se relaciona con la gestión } \\
\text { de recursos humanos, salud, seguridad en el trabajo, } \\
\text { adaptación al cambio y gestión del impacto ambiental } \\
\text { y los recursos naturales. }\end{array}$ \\
\hline $\begin{array}{l}\text { Instituto Ethos de Empresas y yen se requiere diálogo y } \\
\text { Responsabilidad social }\end{array}$ & $\begin{array}{l}\text { Se refiere al público interno, se respeto al } \\
\text { participación, respeto al individuo y respeto } \\
\text { trabajador (atención y cuidado con la salud, seguridad } \\
\text { y condiciones de trabajo). }\end{array}$ \\
\hline $\begin{array}{l}\text { Social Accountability International } \\
\text { (SAI): Norma de Responsabilidad } \\
\text { Social SA 8000. }\end{array}$ & $\begin{array}{l}\text { Contempla la revisión del trabajo infantil, el trabajo } \\
\text { forzoso, la salud, la seguridad, la libertad sindical, la } \\
\text { discriminación, la disciplina, las horas de trabajo y la } \\
\text { compensación. }\end{array}$ \\
\hline $\begin{array}{l}\text { Organización Internacional para la } \\
\text { Normalización o Estandarización } \\
\text { (ISO): ISO 26000 Guía sobre } \\
\text { Responsabilidad Social }\end{array}$ & $\begin{array}{l}\text { Es la buena respuesta y efectivo cumplimiento de los } \\
\text { compromisos con los stakeholders, incluyendo los } \\
\text { empleados. Se contempla: el empleo, dialogo social, } \\
\text { condiciones de trabajo y protección social, salud, } \\
\text { seguridad en el trabajo, y desarrollo humano. }\end{array}$ \\
\hline
\end{tabular}

Fuente: López et al. (2014)

\subsection{Norma ISO 26000}

La norma ISO 26000 es una guía de responsabilidad social y afirma que "el cumplimiento de la ley es un deber fundamental de cualquier organización y una parte esencial de su programa de responsabilidad social. La norma busca promover un entendimiento común de la responsabilidad social, complementando, pero no reemplazando, otras herramientas e iniciativas existentes. Al aplicar la ISO 26000, las organizaciones deben tener en cuenta la diversidad social, ambiental, legal, cultural, política y organizacional, así como las diferencias en las condiciones económicas, y al mismo tiempo ser coherentes con las normas internacionales de comportamiento". 
REVISTA DE LA UNIVERSIDAD DEL ZULIA. 3ª época. Año 11 N 31, 2020 Jhony Ostos \& Antonieta Hamann /// Responsabilidad social empresarial ...160-181

DOI: http://dx.doi.org/10.46925//rdluz.31.12

La norma ISO 26000 contiene siete materias fundamentales de acciones y expectativas relacionadas con la responsabilidad social, las cuales se denominan: Gobernanza de la organización, derechos humanos, prácticas laborales, medio ambiente, prácticas justas de operación, asuntos de consumidores, y participación activa y desarrollo de la comunidad. En el cuadro 2 se pueden apreciar los conceptos de cada una de las materias principales.

Cuadro 2: Conceptos de las materias principales de la norma ISO 26000

\begin{tabular}{|c|l|}
\hline \multicolumn{1}{|c|}{ MATERIAS } & \multicolumn{1}{|c|}{ CONCEPTO } \\
\hline $\begin{array}{l}\text { PRINCIPALES } \\
\text { organización }\end{array}$ & $\begin{array}{l}\text { Es el factor más importante para permitir que una organización } \\
\text { asuma la responsabilidad de los impactos de sus decisiones e integre } \\
\text { la responsabilidad social en toda la organización. Las } \\
\text { organizaciones deben actuar como un medio para comportarse de } \\
\text { una manera socialmente responsable, por lo que deberían tener un } \\
\text { sistema de gobierno organizativo que permita supervisar y poner en } \\
\text { práctica los principios de responsabilidad social }\end{array}$ \\
\hline Derechos humanos & $\begin{array}{l}\text { El reconocimiento y el respeto por los derechos humanos son } \\
\text { esenciales para el estado de derecho, para los conceptos de justicia } \\
\text { social y equidad, y como sostén básico de las instituciones más } \\
\text { esenciales de la sociedad. } \\
\text { Los estados de derecho tienen el deber y la responsabilidad de } \\
\text { respetar, proteger y cumplir los derechos humanos. }\end{array}$ \\
\hline 3. Prácticas laborales & $\begin{array}{l}\text { La creación de empleos, así como los salarios, y otras } \\
\text { compensaciones pagadas por el trabajo realizado, se encuentran } \\
\text { entre las contribuciones económicas y sociales más importantes de } \\
\text { una organización. El trabajo significativo y productivo es esencial en } \\
\text { el desarrollo humano, ya que los niveles de vida se mejoran a través } \\
\text { del empleo pleno y seguro, y su ausencia es una causa primaria de } \\
\text { problemas sociales. Las prácticas laborales socialmente } \\
\text { responsables son esenciales para la justicia social, la estabilidad y la } \\
\text { paz. El medio ambiente }\end{array}$ \\
\hline $\begin{array}{l}\text { La responsabilidad ambiental es una condición previa para la } \\
\text { supervivencia y la prosperidad de los seres humanos. Los asuntos } \\
\text { ambientales están estrechamente vinculados a otros temas de } \\
\text { responsabilidad social. La educación ambiental y el desarrollo de } \\
\text { capacidades es fundamental para promover el desarrollo de } \\
\text { sociedades y estilos de vida sostenibles. (Normas ISO 2600o:sf, pág. } \\
\text { 4l) }\end{array}$ \\
\hline
\end{tabular}


REVISTA DE LA UNIVERSIDAD DEL ZULIA. 3ª época. Año ll N 31, 2020 Jhony Ostos \& Antonieta Hamann /// Responsabilidad social empresarial ...160-181

DOI: http://dx.doi.org/10.46925//rdluz.31.12

\begin{tabular}{|l|l|l|}
\hline $\begin{array}{l}\text { 5. Prácticas justas de } \\
\text { operación }\end{array}$ & $\begin{array}{l}\text { Se refiere a la forma en que una organización utiliza sus relaciones } \\
\text { con otras organizaciones para promover resultados positivos. Los } \\
\text { resultados positivos se pueden lograr proporcionando liderazgo y } \\
\text { promoviendo la adopción de la responsabilidad social de manera } \\
\text { más amplia en toda la esfera de influencia de la organización. }\end{array}$ \\
\hline $\begin{array}{l}\text { 6. Asuntos } \\
\text { consumidores }\end{array}$ & $\begin{array}{l}\text { Los problemas de los consumidores relacionados con la } \\
\text { responsabilidad social son afines, entre otros, a las prácticas de } \\
\text { comercialización justa, protección de la salud y la seguridad, } \\
\text { consumo sostenible, solución de controversias y reparación, } \\
\text { protección de datos y privacidad, acceso a productos y servicios } \\
\text { esenciales, los cuales responden a las necesidades de personas } \\
\text { vulnerables y desfavorecidas. }\end{array}$ \\
\hline $\begin{array}{l}\text { 7. Participación activa y } \\
\text { desarrollo de la } \\
\text { comunidad }\end{array}$ & $\begin{array}{l}\text { La participación activa y desarrollo de la comunidad son partes } \\
\text { integrales del desarrollo sostenible, abarca el apoyo y la } \\
\text { construcción de una relación con la comunidad. La contribución de } \\
\text { una organización al desarrollo de la comunidad puede ayudar a } \\
\text { promover niveles altos de bienestar, mejorando la calidad de vida de } \\
\text { la población. El desarrollo comunitario no es un proceso lineal de } \\
\text { corto plazo sino un proceso a largo plazo en el que están presentes } \\
\text { la satisfacción de las partes interesadas. }\end{array}$ \\
\hline
\end{tabular}

Fuente: Norma ISO 26000.

\subsection{Responsabilidad social empresarial y los resultados financieros}

La participación de las organizaciones en actividades socialmente responsables no solo mejora la satisfacción de las partes interesadas, sino que también tiene un resultado positivo en la reputación de las organizaciones, reduciendo los riesgos financieros (Gras-Gil et al, 2016).

Los autores utilizan diversas medidas para analizar los resultados financieros de las empresas, los más usados son los ingresos, el resultado neto, la rentabilidad sobre activos (ROA), la rentabilidad sobre el capital (ROE), y la Q de Tobin, entre otros. Estudios como de Maqbool y Zameer (2018) utilizan el ROE y el ROA en sus estudios para conocer el impacto de los resultados financieros de la banca comercial en la India. En esta misma línea, RodriguezFernandez (2015) menciona que las empresas se centran en actividades de contenido social pero fijándose siempre en maximizar el rendimiento económico para satisfacer a los accionistas y cumplir con los objetivos trazados, para ello utilizan el ROA, el ROE y la Q de Tobin; demuestran 
REVISTA DE LA UNIVERSIDAD DEL ZULIA. 3ª época. Año ll N 31, 2020 Jhony Ostos \& Antonieta Hamann /// Responsabilidad social empresarial ...160-181

DOI: http://dx.doi.org/10.46925//rdluz.31.12

que las empresas españolas que cotizan en la bolsa de Madrid son rentables y socialmente responsables, lo que hace que estas empresas al aplicar políticas de RSE obtienen mejores resultados financieros.

Otro estudio que utiliza el ROE y el ROA es el de Zoubir (2015), que fue realizado en empresas chinas que cotizan en bolsa, lo resultados muestran que existe una correlación positiva entre la responsabilidad social empresarial y el desempeño financiero. Acar y Kara (2014) mencionan que las actividades de responsabilidad social se han convertido en una importante herramienta corporativa en los últimos años y lo demuestran utilizando los indicadores ROE y ROA. De la misma forma, Mi et al (2018) miden el impacto de la RSE en el desempeño financiero y utilizan la Q de Tobin, el ROE, y el ROA.

\subsection{Great place to work (GPTW)}

El instituto Great Place to Work es una empresa global de investigación, asesoría y capacitación que ayuda a las organizaciones a identificar, crear y mantener excelentes lugares de trabajo a través del desarrollo de culturas de trabajo confiables. Ofrece asistencia a empresas en más de 50 países en los cinco continentes. Sus clientes son empresas y organizaciones que desean crear y sostener excelentes lugares para trabajar, en donde la confianza, la contribución personal y el reconocimiento, permiten crean culturas que facilitan un desempeño organizacional superior. A través del tiempo, la organización Great Place to Work descubrió que la clave para crear un excelente lugar de trabajo no es un conjunto de beneficios, ni programas que se otorgan a los colaboradores, sino la construcción de relaciones caracterizadas por la confianza, el orgullo y el compañerismo. Es así que la confianza deja de ser un factor secundario y se convierte en un factor clave para mejorar los resultados de negocio.

Después de evaluar a muchas empresas localizadas en 50 países del mundo en los 5 continentes, el instituto Great Place to Work selecciona a aquellas organizaciones cuyo desempeño son destacados. La organización posee dos principales herramientas de evaluación: la encuesta de empleados de trust index y el cuestionario de gestión de auditoría cultural. A través de estas encuestas se pretende tener una percepción acerca de cómo se sienten los empleados en el ambiente laboral en el trabajo, a continuación, se explica brevemente en qué consisten cada una de las encuestas. 
REVISTA DE LA UNIVERSIDAD DEL ZULIA. 3ª época. Año ll N 31, 2020 Jhony Ostos \& Antonieta Hamann /// Responsabilidad social empresarial ...160-181

DOI: http://dx.doi.org/10.46925//rdluz.31.12

\subsubsection{La encuesta de empleados de trust index}

Los dos tercios de evaluación de las empresas se realizan a los empleados, lo que se obtiene es información confidencial y anónima por parte de los trabajadores que laboran en cada centro de trabajo, se considera que esta información es la mejor forma de saber si una empresa es un buen lugar para trabajar.

La encuesta explora la satisfacción de los empleados en cinco dimensiones: credibilidad, respeto, justicia, orgullo y camaradería. La credibilidad, se mide por la competencia, la integridad y la comunicación; el respeto, se mide por el apoyo, la colaboración y el cuidado; la justicia, se mide por la equidad y la imparcialidad; la camaradería, se mide por la hospitalidad y la comunidad; y el orgullo, se mide por el trabajo en equipo y la organización de los trabajos personales y grupales.

\subsubsection{El cuestionario de gestión de auditoría cultural}

Un tercio de la evaluación total es representado por este cuestionario. Lo que se recopila es la información sobre el sistema de valores, los programas y las prácticas de la organización. El llenado de esta encuesta es realizado por la misma organización. La auditoría cultural consta de dos partes: Parte I, incluye datos demográficos de los empleados, información general sobre la organización y beneficios ofrecidos a los empleados; Parte II, incluye preguntas abiertas acerca de la filosofía de la empresa, la forma de contratación, comunicación, desarrollo de empleados y celebraciones que se llevan a cabo.

La finalidad de este cuestionario es determinar si la empresa posee buenas prácticas en nueve áreas: contrataciones, inspiración, comunicación, atención, agradecimiento, desarrollo, cuidado, celebraciones y participación en el compartir.

\section{Metodología}

El diseño de esta investigación fue basado en un estudio cualitativo de casos comparativos entre empresas de la muestra, este tipo de investigación es adecuado cuando se requiere identificar las particularidades de un fenómeno que ocurre en contextos cotidianos, y cuando se desea tener conclusiones más integrales que representen a una muestra de casos en 
REVISTA DE LA UNIVERSIDAD DEL ZULIA. 3ª época. Año ll N 31, 2020 Jhony Ostos \& Antonieta Hamann /// Responsabilidad social empresarial ...160-181

DOI: http://dx.doi.org/10.46925//rdluz.31.12

lugar de obtener conclusiones que representen a un solo caso de estudio (Saenz, 2019). El procedimiento de estudio siguió el protocolo de investigación utilizado por Saenz (2019), el cual consistió en una estrategia de investigación de triangulación de múltiples fuentes de evidencia, los cuales sirvieron como medios de corroboración. Las evidencias, fueron obtenidas de información secundaria, y corresponden a fuentes oficiales y públicas, dentro de ellas tenemos a los informes del Instituto Great Place to Work, informes/memoria de las empresas, sitios web, noticias, etc.

La muestra de estudio fueron empresas peruanas que se encontraban reportadas en los informes de clasificación del instituto Great Place to Work de los años 2015, 2016, y 2017. Cada empresa seleccionada fue evaluada tomando en cuenta el cumplimiento de los principios fundamentales de la norma ISO 26000. En cuanto a los resultados financieros, cada empresa fue evaluada tomando en cuenta cuatro indicadores: el ingreso, el resultado neto, el ROA, y el ROE, los datos fueron extraídos de las memorias anuales.

El proceso de selección de las empresas se llevó a cabo de la siguiente forma: primero se tomaron en cuenta las 31 empresas que tenían 1,000 trabajadores o más; segundo, de este total de empresas se seleccionaron 6 de acuerdo a los siguientes criterios: A) las empresas debían haber permanecido en la clasificación del Great Place to Work, durante los tres años del período de estudio; B) las empresas debían poseer estados financieros individuales, es necesario indicar que no todas las empresas tenían estados financieros individuales porque pertenecían a un holding o corporación; C) Los estados financieros debían ser de información pública. La relación final de las empresas que cumplieron con las condiciones fueron las siguientes: Interbank, MAPFRE, Scotiabank, Saga Falabella, SODIMAC, y Supermercados Peruanos.

\section{Resultados}

En esta sección se presenta la información clave que corresponde a la participación de cada una de ellas en las acciones de Responsabilidad social y los resultados financieros obtenidos en el período de estudio.

\section{-INTERBANK}


REVISTA DE LA UNIVERSIDAD DEL ZULIA. 3ª época. Año ll N 31, 2020 Jhony Ostos \& Antonieta Hamann /// Responsabilidad social empresarial ...160-181

DOI: http://dx.doi.org/10.46925//rdluz.31.12

Es una de las principales instituciones financieras del país y a la fecha posee más de 2 millones de clientes. Ha tenido un desempeño financiero sostenido por lo que las tres principales clasificadoras de riesgo lo calificaron como una empresa con buen grado de inversión (Interbank, 2018). Interbank participa en diversos proyectos de responsabilidad social empresarial, a la fecha el banco se ha involucrado en la construcción de proyectos de mejoramiento del tránsito vehicular y la construcción de puentes vehiculares. En total se ha calculado que los proyectos benefician a un aproximado de 877 mil ciudadanos (Interbank, 2018).

El Gerente General de la empresa, Luis Felipe Castellanos indicó que Interbank "posee una cultura que valora el aporte de todos y está abierto a escuchar opiniones diferentes, es una organización donde puedes crecer profesional y personalmente" (Great Place to Work (GPTW), 2018, p.32).

\section{-MAPFRE}

Es una empresa aseguradora global, la cual está considerada como posición de referencia en los principales mercados mundiales (Mapfre, 2017). Por ejemplo, está clasificada

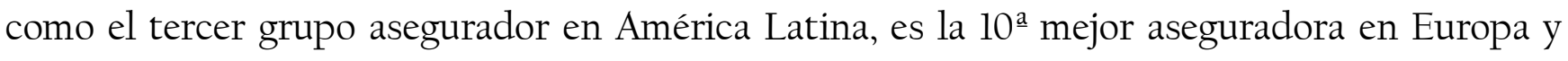
pertenece al TOP 20 de seguros de automóvil en Estados Unidos. Para MAPFRE la confianza es un valor muy apreciado. Renzo Calda, Gerente General de Mapfre Perú, señala que "a través de la transparencia en nuestros procesos y servicios, Mapfre ha logrado ganarse la confianza de sus colaboradores y clientes externos e internos" (GPTW, 2018, p.36).

\section{-SCOTIABANK}

Se posicionó en el Perú e inició sus operaciones en el año 2006. Scotiabank forma parte de The Bank of Nova Scotia (BNS), es el banco canadiense con mayor prestigio internacional, y una de las instituciones financieras más importantes de Norteamérica. Dentro de sus numerosos logros se encuentra el haber sido reconocido como el $3^{\circ}$ banco más importante de Canadá y el $24^{\circ}$ más importante del mundo en el 2014. A su vez, hacia fines del 2014 declaró poseer activos consolidados por más de 852,000 mil millones de dólares canadienses. En el Perú, Scotiabank fue el primer banco en obtener por dos anDos consecutivos (2012 y 2013) el distintivo de empresa socialmente responsable (ESR), dicha distinción fue otorgada por la Asociación Peru[ 2021. Scotiabank está comprometido con el desarrollo del país, por lo que sus acciones se guían con 
REVISTA DE LA UNIVERSIDAD DEL ZULIA. 3ª época. Año ll N 31, 2020 Jhony Ostos \& Antonieta Hamann /// Responsabilidad social empresarial ...160-181

DOI: http://dx.doi.org/10.46925//rdluz.31.12

los principios internacionales del pacto mundial, con la carta de las empresas para un desarrollo sostenible de la cámara de comercio internacional, y acuerdos nacionales como la asociación de buenos empleadores (Scotiabank, sf).

Miguel Uccelli, Gerente General de Scotiabank, afirmó que una de las principales fortalezas del banco es ser "percibidos como una empresa inclusiva y diversa, que le brinda un mundo de posibilidades a todos y todas, y donde el empoderamiento de la mujer es una realidad" (GPTW, 2018, p.33).

\section{-SAGA FALABELLA}

Saga Falabella es una tienda por departamentos considerada como una de las más grandes y consolidadas de América Latina. En la actualidad, Falabella es considerada como la empresa más importante de Sudamérica en su rubro y se encuentra posicionada en el Perú, Chile, Argentina y Colombia. La empresa tiene una gran actividad de responsabilidad social, la empresa se ha involucrado en diversos proyectos, tales como, haciendo escuela en el cual se apadrinaron nueve escuelas y se benefició a un total de 9,739 alumnos, también participó en el programa sembrando vida, participando en el sembrado de 29 árboles en el colegio y en la casa de retiro de la institución Fe y Alegría. Adicionalmente, en las tiendas de Saga Falabella se incorporaron distintos espacios destinados a la recolección de fondos para la lucha contra el cáncer (Falabella, 2018).

Alex Zimmermann, Gerente General de Saga Falabella, afirmó que el liderazgo colaborativo de la empresa es la clave de su éxito. Señala, "en Saga Falabella hemos aprendido a valorar la diversidad de opiniones dentro de la organización" (GPTW, 2018, p.34).

\section{-SODIMAC}

Sodimac es una empresa líder en el rubro retail e industria. Dentro de las actividades que realiza se encuentra el proveer soluciones a los proyectos de construcción, y el contribuir en el mejoramiento y en la decoración de los hogares. En el año 2017 obtuvo un resultado de 57\% en el indicador de sostenibilidad, el cual lo posicionó 5 puntos arriba respecto del año anterior (Sodimac Perú, 2017). En cuanto a la Responsabilidad Social empresarial, SODIMAC realiza una evaluación anual de su desempeño a través de un indicador estratégico que emplea los siguientes instrumentos internacionales: principios del pacto mundial de las naciones unidas, directrices 
REVISTA DE LA UNIVERSIDAD DEL ZULIA. 3ª época. Año ll N 31, 2020 Jhony Ostos \& Antonieta Hamann /// Responsabilidad social empresarial ...160-181

DOI: http://dx.doi.org/10.46925//rdluz.31.12

para empresas multinacionales de la OCDE, ISO 26.000, Global Reporting Initiative (GRI), principios rectores empresas y derechos humanos de las naciones unidas, e índice de sostenibilidad Dow Jones (DJSI).

Hernán Pérez, Gerente General de Sodimac Perú y Maestro Perú, afirmó que la empresa ha decidido incorporar en el grupo de asesores a adultos mayores, con capacidades, culturas e intereses diferentes. Así pues, señala que "Nuestra riqueza se encuentra en la diversidad y la experiencia" (GPTW, 2018, p. 35).

\section{-SUPERMERCADOS PERUANOS}

Supermercados Peruanos pertenece a la corporación Intercorp y a la fecha engloba a los supermercados: Vivanda, Plaza Vea y Mass. En un inicio, Supermercados Peruanos operaba bajo el nombre de Supermercados Santa Isabel S.A. hasta que, en el 2003, el grupo financiero Interbank adquirió la totalidad de sus acciones brindando a la empresa el respaldo financiero y el prestigio que hoy día posee. En cuanto a sus acciones de responsabilidad social corporativa, Supermercados Peruanos fomenta la educación de primer nivel mediante las donaciones que recauda de los clientes en cada una de sus tiendas, de este modo, cientos de niños con escasos recursos pueden acceder a una educación de calidad. El financiamiento de los estudios se da a través de becas otorgadas por la asociación civil sin fines de lucro PERU CHAMPS. Asimismo, Supermercados Peruanos apoya a la Teletón ofreciendo sus instalaciones como centro de recaudación de donaciones voluntarias (Supermercados Peruanos, 2019).

Juan Carlos Vallejo, CEO de Supermercados Peruanos, señala que el liderazgo existente en la empresa es la base fundamental del éxito sostenido, sostiene que en Supermercados Peruanos "se impulsa un liderazgo transformacional basado en el modelo de competencias, que busca líderes determinados que sean capaces de movilizar a los equipos, centrados en el cliente, que tomen decisiones, y que promuevan siempre un entorno colaborativo" (GPTW, 2018, p.32)

Se analizaron los resultados financieros de cada una de las empresas de la muestra y fueron comparados con el año base 2014. En la tabla 1 se puede observar los resultados obtenidos, el signo bien significa que el resultado financiero de ese año fue mejor que el año 2014. También 
REVISTA DE LA UNIVERSIDAD DEL ZULIA. 3ª época. Año ll N 31, 2020 Jhony Ostos \& Antonieta Hamann /// Responsabilidad social empresarial ...160-181

DOI: $\underline{\text { http://dx.doi.org/10.46925//rdluz.31.12 }}$

se aprecia que existe una tendencia de mejores resultados en los ingresos y en el resultado neto. Sin embargo, el ROA Y ROE tuvieron comportamientos variados.

En el anexo 1 (cuadro 3) se observan las actividades de responsabilidad social que cumplieron cada empresa en cada una las materias fundamentales de la norma ISO 26000. Se aprecia también que cada empresa tuvo una actividad destacada en el cumplimiento de los requerimientos de la norma ISO 26000, cuyas actividades fueron soportadas por un sistema de gobierno organizativo que supervisa y pone en práctica los principios de responsabilidad social.

\section{Discusión}

El objetivo del estudio fue examinar las prácticas de responsabilidad social y analizar los resultados financieros obtenidos de una muestra de las mejores empresas para trabajar (GPTW, período 2015 - 2017) de un mercado emergente. En cuanto al análisis de las prácticas de RSE, se observó que las empresas ejecutaron y desarrollaron diversas actividades que encajaban con los requerimientos de la norma ISO 26000; también se puede afirmar que las empresas desarrollan diversas prácticas de responsabilidad social, las cuales son originales y a la vez no homogéneas entre las empresas, probablemente por la desigual exigencia y valoración de la RSE de las partes interesadas que se encuentran en cada tipo de industria.

Respecto de los resultados financieros, se observó que existe una tendencia a la obtención de resultados financieros positivos, los cuales se pueden apreciar con mayor contundencia en los ingresos y en los resultados netos anuales, no pasa lo mismo con el ROE y ROA, las cuales, al estar vinculados con las cuentas del balance general (activo, pasivo, patrimonio) tienen resultados financieros diversos que podrían ser analizados en un posterior estudio.

Se observa también que los resultados financieros de corto plazo son prioridad, es necesario que las empresas encuentren un equilibrio en la ejecución de las actividades de RSE y en el beneficio social. Al respecto (Muscalu et al., 2012) sostienen que la implementación de acciones de responsabilidad social requiere esfuerzos considerables por parte de las empresas, y sin duda, los beneficios que obtendrán se multiplicarán 
REVISTA DE LA UNIVERSIDAD DEL ZULIA. 3a época. Año $11 \mathrm{~N}^{\circ}$ 31, 2020 Jhony Ostos \& Antonieta Hamann /// Responsabilidad social empresarial ...160-181

DOI: http://dx.doi.org/10.46925//rdluz.31.12

Tabla 1. Resultados financieros de las mejores empresas para trabajar (GPTW)

\begin{tabular}{|c|c|c|c|c|c|}
\hline \multirow{2}{*}{ Empresas } & \multirow{2}{*}{ Años } & \multicolumn{4}{|c|}{$\begin{array}{l}\text { Resultados financieros } \\
\text { (respecto año 2014) }\end{array}$} \\
\hline & & Ingresos & $\begin{array}{l}\text { Resultado } \\
\text { Neto }\end{array}$ & ROA & ROE \\
\hline \multirow{3}{*}{ Interbank } & 2015 & $\checkmark$ & $\checkmark$ & & $\checkmark$ \\
\hline & 2016 & $\checkmark$ & $\checkmark$ & & \\
\hline & 2017 & $\checkmark$ & $\checkmark$ & & \\
\hline \multirow{3}{*}{ Mapfre } & 2015 & $\checkmark$ & $\checkmark$ & & \\
\hline & 2016 & $\checkmark$ & $\checkmark$ & $\checkmark$ & $\checkmark$ \\
\hline & 2017 & $\checkmark$ & $\checkmark$ & & \\
\hline \multirow{3}{*}{ Scotiabank } & 2015 & $\checkmark$ & $\checkmark$ & & \\
\hline & 2016 & $\checkmark$ & $\checkmark$ & $\checkmark$ & \\
\hline & 2017 & $\checkmark$ & $\checkmark$ & $\checkmark$ & \\
\hline \multirow{3}{*}{ Saga Falabella } & 2015 & $\checkmark$ & $\checkmark$ & $\checkmark$ & $\checkmark$ \\
\hline & 2016 & $\checkmark$ & $\checkmark$ & & $\checkmark$ \\
\hline & 2017 & $\checkmark$ & $\checkmark$ & & $\checkmark$ \\
\hline \multirow{3}{*}{ Sodimac } & 2015 & $\checkmark$ & & & $\checkmark$ \\
\hline & 2016 & $\checkmark$ & & & \\
\hline & 2017 & $\checkmark$ & & & \\
\hline \multirow{3}{*}{$\begin{array}{l}\text { Supermercados } \\
\text { Peruanos }\end{array}$} & 2015 & $\checkmark$ & $\checkmark$ & $\checkmark$ & $\checkmark$ \\
\hline & 2016 & $\checkmark$ & $\checkmark$ & $\checkmark$ & $\checkmark$ \\
\hline & 2017 & $\checkmark$ & $\checkmark$ & $\checkmark$ & $\checkmark$ \\
\hline
\end{tabular}

Nota: Compilado por los autores, modelo de análisis adaptado de Saenz \& Brown (2018)

Fuente: Estados financieros de cada empresa

En adición, se podría discutir que la selección de la muestra ayudó en la obtención de los resultados positivos, sin embargo, esto no invalida los resultados del estudio, porque las empresas fueron calificadas por sus trabajadores como mejores empresas para trabajar sin tomar en cuenta las acciones de responsabilidad social realizadas por estas.

\section{Conclusiones}

El estudio fue realizado para una muestra de las mejores empresas para trabajar en el Perú obtenidos en el listado del instituto del Great place to work, se puede concluir que, A) las 
REVISTA DE LA UNIVERSIDAD DEL ZULIA. 3ª época. Año ll N 31, 2020 Jhony Ostos \& Antonieta Hamann /// Responsabilidad social empresarial ...160-181

DOI: http://dx.doi.org/10.46925//rdluz.31.12

empresas desarrollan actividades de RSE y cumplen con los lineamientos de la norma ISO 26000; B) las actividades de RSE que son implementadas por las empresas, en muchos casos, son actividades adicionales y no corresponden a su principal propuesta de valor; C) Las empresas priorizan los resultados financieros de corto plazo (ingresos y resultado neto anuales; D) los resultados financieros de corto plazo permiten sostener los fondos necesarios para realizar las prácticas de RSE.

Los hallazgos obtenidos no podrían ser generalizados a todo tipo de empresas, por lo que en futuras investigaciones se podría extender el estudio a organizaciones que no se encuentran dentro de este grupo de estudio. De otro lado, las industrias no son homogéneas en la exigencia ni valoración de las prácticas de responsabilidad social, por lo que también sería necesario extender el estudio por tipos de industria, es decir: manufactura, servicios, minería, construcción, y otros.

En el estudio se encontró que las mejores empresas para trabajar mantienen resultados financieros positivos principalmente de corto plazo, los académicos y gerentes tendrían que continuar explorando los beneficios no financieros, más allá que solo el beneficio financiero. En adición, proponemos como futuro estudio, averiguar cuál de las prácticas de RSE tienen mayor significancia y/o valoración por las partes interesadas y por la sociedad.

\section{Referencias}

Acar, D., y Kara, E. (2014). Analyzing the effects of corporate social responsibility level on the financial performance of companies: An application on bist corporate governance index included companies. International Journal of Management Economics and Business, 10(23), 227-242, doi: 2147-9208 E-ISSN:2147-9194

Barić, A. (2017). Corporate social responsibility and stakeholders: Review of the last decade (2006-2015). Business Systems Research: International journal of the Society for Advancing Innovation and Research in Economy Journal, 8(1), 133-146, doi:10.1515/bsrj-2017-0011

Edelman. (2017). 2017 Edelman trust barometer. Recuperado de https://www.edelman.com/trust2017/.

Falabella Perú. (2018). Reporte de Sostenibilidad Corporativa 2018. Recuperado de https://www.falabella.com.pe/static/staticContent/content/minisitios/Inversionistas/pdf/Anex o_Reporte_Sostenibilidad_2018.pdf 
REVISTA DE LA UNIVERSIDAD DEL ZULIA. 3a época. Año $11 \mathrm{~N}^{\circ}$ 31, 2020 Jhony Ostos \& Antonieta Hamann /// Responsabilidad social empresarial ...160-181

DOI: http://dx.doi.org/10.46925//rdluz.31.12

Godos, J., Cabeza, L., Martínez, D., y Fernández, R. (2018). Factors influencing board of directors' decision-making process as determinants of CSR engagement. Review of Managerial Science, 12(1), 229-253. doi:10.1007/s11846-016-0220-1

Gras-Gil, E., Palacios, M. y Hernández, J. (2016). Investigating the relationship between corporate social responsibility and earnings management: Evidence from Spain. BR2 Business Research Quarterly, 19(1), 289-299, doi: 10.1016/j.brq.2016.02.002

Great place to work. (2018). Mejores lugares para trabajar 2018. Recuperado de https://www.greatplacetowork.com.pe/los-mejores-lugares-para-trabajar/las-mejores/2018

Hohnen, P. (2007). Corporate Social Responsibility. An Implementation Guide for Business. In: "International Institute for Sustainable Development". Recuperado de https://www.iisd.org/pdf/2007/csr_guide.pdf

Interbank. (2018). Reporte de Sostenibilidad Interbank 2018. Recuperado de https://interbank.pe/documents/20182/2263274/reporte-de-sostenibilidad-2018.pdf/44b35dfcd449-466a-ab6c-0bab6db02cd3

Jaramillo, O. L. (2011). La dimensión interna de la responsabilidad social en las micro, pequeñas y medianas empresas del programa expopyme de la Universidad del Norte. Pensamiento é gestión, (31), 167-195.

López, V., Moreno, L., y Marín, M. (2014). La responsabilidad social interna, estudio analítico para las plantas maquiladoras. Investigación Administrativa, 43(114), 23-42.

Mapfre. (2017). Informe Integrado 2017. Recuperado de https:/www.mapfre.com/media/sostenibilidad/2017/informe-integrado-mapfre-2017.pdf

Maqbool, S., y Zameer, M. (2018). Corporate social responsibility and financial performance: An empirical analysis of Indian banks. Future Business Journal, 4(1), 84-93. doi: 10.1016/j.fbj.2017.12.002

Martínez de Carrasquero, C. (2019). Responsabilidad social universitaria, transferencia tecnológica y desarrollo endógeno. Estrategias de vinculación comunitaria, Revista Latinoamericana de Difusión Científica, 1 (l), 55-67. http://difusioncientifica.info/index.php/difusioncientifica/article/view/5

Mi, J., Jiang, S., Tao, X., y Hu, W. (2018). The relevance of social responsibility and financial performance of listed companies. Journal of Management and Sustainability, 8 (2), 40-50.

Muscalu, E., Fraticiu, L., y Ghitulete, A. (2012). Corporate social responsability in the current global economic context. Review of general management, 15(1), pp. 85-92. 
REVISTA DE LA UNIVERSIDAD DEL ZULIA. 3a época. Año $11 \mathrm{~N}^{\circ}$ 31, 2020 Jhony Ostos \& Antonieta Hamann /// Responsabilidad social empresarial ...160-181

DOI: http://dx.doi.org/10.46925//rdluz.31.12

Normas ISO 26000 (s.f). Norma internacional ISO 26000, Guía sobre responsabilidad social. Recuperado de https://www.normas-iso.com/iso-26000-responsabilidad-social/

Park, B., Chidlow, A., y Choi, J. (2014). Corporate social responsibility: Stakeholders influence on MNEs' activities. International Business Review, 23(5), 966-980, doi.org/10.1016/j.ibusrev.2014.02.008

Rezaee, Z. (2017). Corporate Sustainability: Theoretical and Integrated Strategic Imperative and Pragmatic Approach. The Journal of Business Inquiry, 16(1). Recuperado de https://ssrn.com/abstract=3148705

Rodriguez-Fernandez, M. (2015). Social responsibility and financial performance: The role of good corporate governance. BR2 Business Research Quarterly, 19 (29), 137-151.

Saenz, C. (2019). Earning a social license to operate in mining: A case study from Peru. Resources Policy, 64, https://doi.org/10.1016/j.resourpol.2019.101482

Saenz, C. y Brown, H. (2018). The disclosure of anticorruption aspects in companies of the construction sector: Main companies worldwide and in Latin America. Journal of cleaner production, 196, 259-272.

Scandelius, C., y Cohen, G. (2016). Achieving collaboration with diverse stakeholders-The role of strategic ambiguity in CSR communication. Journal of Business Research,69(9), 3487-3499. doi:10.1016/j.jbusres.2016.01.037.

Schmitz, J. y Schrader, J. (2015). Corporate Social Responsibility: A Microeconomic Review of the Literature. Journal of Economic Surveys, 29(1), 27-45. https://doi.org/10.11l1/joes.12043

Scotiabank (s.f.). Brochure institucional de Responsabilidad Social Corporativa. Recuperado de https:/www.scotiabank.com.pe/rss-sbp/descarga/brochure.pdf

Sodimac (2017). Reporte de sostenibilidad 2017. Recuperado de https://www.sodimac.com.pe/static/categorias/contenidoEstatico/masdesodimac/responsabili dad-social/pdf/Reporte\%202017-SODIMAC-Completo.pdf?cid=homhom29963

Su, C., Lee, E. M., y Lee, Y. (2019). An empirical research of environment management strategy: Exploring the relationships among perceived corporate social responsibility, organizational trust, perceived external prestige and organizational identification. International Journal of Organizational Innovation, 12(1), 245-260.

Supermercados Peruanos (2019). Reporte de sostenibilidad 2018. Recuperado de https://www.bvl.com.pe/hhii/OE5087/20190315093601/REPORTE32SOSTENIBILIDAD32PAR TE32B322018324532INRETAIL.PDF 
REVISTA DE LA UNIVERSIDAD DEL ZULIA. 3ª época. Año ll N 31, 2020 Jhony Ostos \& Antonieta Hamann /// Responsabilidad social empresarial ...160-181

DOI: http://dx.doi.org/10.46925//rdluz.31.12

Taghian, M., D'Souza, C., y Polonsky, M. (2015). A stakeholder approach to corporate social responsibility, reputation and business performance. Social Responsibility Journal, 11(2), 340-363. https://doi.org/10.1108/SRJ-06-2012-0068

Valiorgue, B., Acquier,A., y Daudigeos, T. (2017). Sharing the Shared Value: A Transaction Cost Perspective on Strategic CSR Policies in Global Value Chains. Journal of Business Ethics, 144, 139152. https://doi.org/10.1007/s10551-015-2820-0

Vashchenko, M. (2017). An external perspective on CSR: What matters and what does not?. Business Ethics: A European Review, 26(4), 396-412. https://doi.org/10.1111/beer.12162

Woodroof, P., Deitz, G., Howie, K., y Evans, R. (2019). The effect of cause-related marketing on firm value: a look at Fortune's most admired all-stars. Journal of the Academy of Marketing Science, 47, 899-918. Recuperado de https://ink.springer.com/article/10.1007/sll747-019-00660-y

Zoubir, F. (2015). Corporate social responsibility and financial performance. Accounting, Auditing and Finance, 4(1), 74-85. 
REVISTA DE LA UNIVERSIDAD DEL ZULIA. 3a época. Año $11 \mathrm{~N}^{\circ}$ 31, 2020 Jhony Ostos \& Antonieta Hamann /// Responsabilidad social empresarial ...160-181 DOI: http://dx.doi.org/10.46925//rdluz.31.12

Cuadro 3. Acciones de responsabilidad social según la norma ISO 26000

\begin{tabular}{|c|c|c|c|}
\hline & INTERBANK & MAPFRE & SAGA FALABELLA \\
\hline DERECHOS HUMANOS & $\begin{array}{l}\text { Promueven la no discriminación yel respeto } \\
\text { de los Derechos Humanos } \\
\text { En la selección de personal se priorizan los } \\
\text { valores } \\
\text {-Creencia en la igualdad de los derechos y } \\
\text { oportunidades de las personas, la gestión } \\
\text { descentralizada a porta la inclusión } \\
\text { Creen en el equilibrio de genero, el } \\
\text { personal femenino es alrededor del } 60 \% \text {, el } \\
29 \% \text { de los cargos de alta gerencia son } \\
\text { ocupados por mujeres }\end{array}$ & $\begin{array}{l}\text {.Se promueve la inclusión de personas con } \\
\text { discapacidad, propiciando la igualdad de } \\
\text { oportunidades } \\
\text {.Diseño de oficinas de atención al público } \\
\text { adaptadas para personas con discapacidad. } \\
\text {.Protocolo para la Prevención y tratamiento del } \\
\text { Acoso en la empresa. } \\
\text {.Formación del personal en valores y políticas } \\
\text { globales: Código ético, Igualdad, } \\
\text { responsabilidad social, control Interno,y } \\
\text { discapacidad }\end{array}$ & $\begin{array}{l}\text {.Aplicación del programa de equidad de } \\
\text { género } \\
\text {.Implementación del programa de } \\
\text { inserción laboral para personas con } \\
\text { habilidades especiales } \\
\text {.Aplicación del programa de inserción } \\
\text { laboral para personas mayores de } 60 \\
\text { años } \\
\text {.Aplicación del Programa de Inserción } \\
\text { Laboral de Mujeres en condición de } \\
\text { vulnerabilidad (en conjunto con las } \\
\text { organizaciones Comex Perú y Fe y Alegría) }\end{array}$ \\
\hline PRACTICAS LABORALES & $\begin{array}{l}\text {.Impulso de la universidad corporativa del } \\
\text { grupo - capacitaciones varias y programa de } \\
\text { formación de líderes } \\
\text {.Capacitación presencial y virtual } \\
\text {.Ejecución de programas para reforzar la } \\
\text { cultura y liderazgo } \\
\text {.Servicios varios: Evento "navidad del niño", } \\
\text { programa plan móvil } \\
\text {.Tipo de cambio preferencial y tasas } \\
\text { preferenciales de tarjetas de crédito para } \\
\text { trabajadores } \\
\text {.Productos financieros para trabajadores }\end{array}$ & $\begin{array}{l}\text { PAS: Programa de Asesoría Psicológica, tiene } \\
\text { como objetivo fomentar el cuidado de la salud } \\
\text { mental y el equilibrio de la vida personal con } \\
\text { vida profesional } \\
\text {.Programa de vida saludable que ayuda a los } \\
\text { trabajadores sentirse mejor en armonía con el } \\
\text { trabajo } \\
\text {.Plan de formación comercial y técnica } \\
\text {.Plan de formación e inducción para nuevos } \\
\text { colaboradores } \\
\text {.Programa de educación, formación, } \\
\text { asesoramiento, prevención y control de riesgos }\end{array}$ & $\begin{array}{l}\text {.Programa liderando el talento } \\
\text {.Escuela de jefes } \\
\text { Programa de mentores } \\
\text {.Capacitación en productos, e-learning y } \\
\text { presencial } \\
\text {.Creciendo juntos, cajeros y vendedores } \\
\text { entrenados } \\
\text {.Campaña de valores y buenas prácticas }\end{array}$ \\
\hline MEDIO AMBIENTE & $\begin{array}{l}\text { Proyecto Paper-Less, programa de } \\
\text { digitalización y reducción de materiales, es una } \\
\text { iniciativa que busca convertir al banco en } \\
\text { una empresa más digital con menos } \\
\text { consumo de recursos físicos. } \\
\text { Se pone énfasis en el uso de tecnología para } \\
\text { reducir el consumo energético en las tiendas } \\
\text { Financieras, canales Electrónicos y sedes } \\
\text { Administrativas. }\end{array}$ & $\begin{array}{l}\text { Reciclaje de papel: Concientización del cuidado } \\
\text { del medio ambiente. Generación de conciencia } \\
\text { sobre la importancia de reciclar papel y crearel } \\
\text { hábito para el uso responsable de los recursos } \\
\text { naturales yel medio ambiente. } \\
\text { Implementaciones de mejoras en eficiencia } \\
\text { energética en las oficinas }\end{array}$ & $\begin{array}{l}\text { Campaña de reciclaje, soy F soy Verde } \\
\text { Instauración de la politica de medio } \\
\text { ambiente, respeto a la comunidad, } \\
\text { respeto a biodiversidad yecosistema } \\
\text { Adaptacion al cambio climatico con } \\
\text { gestion de riesgos de desastres } \\
\text { Infraestructura sostenible }\end{array}$ \\
\hline $\begin{array}{l}\text { PRACTICAS JUSTAS DE } \\
\text { OPERACION }\end{array}$ & $\begin{array}{l}\text { Código de ética y conducta de Proveedores, } \\
\text { derechos humanos, condiciones laborales, } \\
\text { conflictos de interés, anticorrupción, } \\
\text { seguridad y medio ambiente, } \\
\text { confidencialidad, competencia yel uso } \\
\text { correcto de activos y de la propiedad } \\
\text { intelectual. }\end{array}$ & $\begin{array}{l}\text { Norma de Compras } \\
\text { Código Ético y de Conducta. } \\
\text { Protocolo de selección de colaboradores. } \\
\text { Control del cumplimiento fiscal, tributario y } \\
\text { laboral de proveedores }\end{array}$ & $\begin{array}{l}\text { - Eleccion Libre del empleo } \\
\text { - Libertad de asociacion yel derecho a } \\
\text { negociaciones colectivas } \\
\text {. Condiciones de trabajo seguras e } \\
\text { higienicas } \\
\text {. No empleo de mano de obra infantil } \\
\text {. Pago de un salario digno } \\
\text {. Horas de trabajo no excesivas } \\
\text {. No discriminacion, no tratro inhumano o } \\
\text { severo }\end{array}$ \\
\hline $\begin{array}{c}\text { ASUNTOS } \\
\text { CONSUMIDORES }\end{array}$ & $\begin{array}{l}\text {. Código de buenas prácticas, implementado } \\
\text { por la Asociación de Bancos del Perú } \\
\text {. Implementacion de procesos internos para } \\
\text { proteger la información de clientes. } \\
\text {. Calidad de información de productos y } \\
\text { servicios, instructivo de publicidad para } \\
\text { comunicación externa } \\
\text { - Cumplimiento de normativa de publicidad } \\
\text { INDECOPI } \\
\text {. Código de protección al Consumidor } \\
\text { Reglamento de transparencia de la } \\
\text { información } \\
\text {. Compromiso del banco para utilizar un } \\
\text { lenguaje simple } \\
\text {. Atención de consultas, quejas, y reclamos, } \\
\text { en forma presencial, banca Telefónica, o } \\
\text { redes sociales }\end{array}$ & $\begin{array}{l}\text { Establecimiento de diversos canales de } \\
\text { comunicación con los clientes, en forma } \\
\text { presencial y a través de redes sociales. } \\
\text { - Se cuenta con un programa de captación y } \\
\text { fidelización de clientes. } \\
\text { - Se realizan acciones específicas para prevenir } \\
\text { intentos de fraude por parte de los asegurados. } \\
\text {. Procedimientos específicos para garantizar la } \\
\text { protección de datos de los clientes. } \\
\text { - Formación de empleados dedicados a la } \\
\text { gestión de la calidad } \\
\text { Unidad orgánica interno de protección al } \\
\text { cliente }\end{array}$ & $\begin{array}{l}\text { Servicio al Cliente via pagina web } \\
\text { Servicio al Cliente via Telefonica } \\
\text { Servicio al cliente en las tiendas fisicas }\end{array}$ \\
\hline $\begin{array}{l}\text { PARTICIPACION ACTIVA Y } \\
\text { DESARROLLO DE LA } \\
\text { COMUNIDAD }\end{array}$ & $\begin{array}{l}\text { - Implementación de tiendas en zonas no } \\
\text { atendidas } \\
\text { - Implementación de programas de } \\
\text { voluntariado } \\
\text {. Apoyo en construcción de comisarias } \\
\text { Programa maestro que deja huella } \\
\text {. Programa techo Perú }\end{array}$ & $\begin{array}{l}\text { - Programa voluntariado } \\
\text {. Día mundial del donante: Mapfre invita a sus } \\
\text { empleados en ésta iniciativa en beneficio de los } \\
\text { niños con cáncer. } \\
\text { - MAPFRE Perú otorgó un seguro gratuito al } \\
\text { Cuerpo General de Bomberos Voluntarios del } \\
\text { Perú como agradecimiento a } 156 \text { años de apoyo a } \\
\text { la comunidad. La póliza permitirá proteger a } \\
\text { todos los bomberos voluntarios que fallezcan o } \\
\text { sufran invalidez en el trabajo. Se otorgarán } \\
\text { indemnizaciones de hasta S/.200.000. Esta no } \\
\text { solo aplicará para incendios sino para todo tipo } \\
\text { de incidentes, tales como accidentes de autoso } \\
\text { desastres naturales. }\end{array}$ & $\begin{array}{l}\text {. Programa Haciendo escuela (Fe y } \\
\text { Alegria) } \\
\text {. Participación en la Teleton } \\
\text { - Apoyo a la comunidad en desastres } \\
\text { naturales }\end{array}$ \\
\hline
\end{tabular}

Fuente: Informes oficiales de Great place to work y memorias de cada empresa 
REVISTA DE LA UNIVERSIDAD DEL ZULIA. 3a época. Año $11 \mathrm{~N}^{\circ}$ 31, 2020 Jhony Ostos \& Antonieta Hamann /// Responsabilidad social empresarial ...160-181 DOI: http://dx.doi.org/10.46925//rdluz.31.12

Cuadro 3. Acciones de responsabilidad social según la norma ISO 26000

\begin{tabular}{|c|c|c|c|}
\hline & SCOTIANBANK & SODIMAC & SUPERMERCADOS PERUANOS \\
\hline DERECHOS HUMANOS & $\begin{array}{l}\text {.Existe una equidad remunerativa en } \\
\text { promedio de los sueldos y salarios del } \\
\text { personal femenino y masculino. } \\
\text {-La diferencia remunerativa del personal, } \\
\text { porgénero, es de } 1 \% \text { ( } 51 \% \text { en varones vs. } 49 \\
\% \text { en mujeres). }\end{array}$ & $\begin{array}{l}\text {.Integración de la cultura de trabajo saludable } \\
\text { Operación bajo los principios de la norma ISO } \\
26000 \\
\text {.Desempeño de acuerdo a los principios de la } \\
\text { Declaración Universal de Derechos Humanos y } \\
\text { la Declaración de la Organización Internacional } \\
\text { del Trabajo (OIT) } \\
\text {-Renovación permanentemente del compromiso } \\
\text { con la ética y transparencia } \\
\text {.Administración del portal web de atención de } \\
\text { consultas y reclamos }\end{array}$ & $\begin{array}{l}\text { Participación en el programa Perú } \\
\text { responsable del Ministerio de trabajo } \\
\text {.Brinda oportunidades laborales a personas } \\
\text { con habilidades especiales } \\
\text {.Participación en la Asociación de buenos } \\
\text { empleadores (ABE) } \\
\text { "Promueve la Responsabilidad Social } \\
\text { Laboral." }\end{array}$ \\
\hline PRACTICAS LABORALES & $\begin{array}{l}\text { Respeto de protección regulada, beneficios } \\
\text { laborales } \\
\text {.Beneficios financieros en productos del } \\
\text { banco } \\
\text {.Beneficios en balance vida-trabajo } \\
\text {.Programas de capacitación } \\
\text { Programas de capacitación in-house,e- } \\
\text { learning } \\
\text { Scotianbank café }\end{array}$ & $\begin{array}{l}\text {.El lanzamiento de programa de beneficios con } \\
4 \text { dimensiones: calidad de vida, ahorro, unidos, } \\
\text { y progreso. Se busca mejorarla calidad de vida } \\
\text { de los empleados } \\
\text {-Incorporación de horario flexible, horas de } \\
\text { licencia de pre y post maternidad } \\
\text {-Renovación del programa de capacitación } \\
\text {-Renovación de Eticacción y canal de denuncias. } \\
\text {.Primera evaluación de sueldos. } \\
\text {-Renovación del voluntariado. } \\
\text { - Inventario de gas de efecto invernadero }\end{array}$ & $\begin{array}{l}\text {.CreSer, herramienta de gestión para elaborar } \\
\text { el plan anual de capacitación, medirel } \\
\text { desempeño, la motivación, y plantear una } \\
\text { línea de carrera. } \\
\text {.Academia SPSA, es una plataforma de } \\
\text { capacitación para alinearlas habilidades } \\
\text { del personal con los objetivos de la empresa } \\
\text {.Programa de trainees dirigido a } \\
\text { colaboradores con potencial, se diseña e } \\
\text { implementa un plan de desarrollo con apoyo } \\
\text { de un gerente o sub gerente } \\
\text {.Administración central: cursos, talleres, y } \\
\text { coaching para los colaboradores con la } \\
\text { finalidad de generarmayorvaloren la } \\
\text { gestión de negocios } \\
\text {.Programa de líderes ambientales, se } \\
\text { capacita ysensibiliza a los colaboradores } \\
\text { para que sean responsables del medio } \\
\text { ambienteysostenibilidad }\end{array}$ \\
\hline MEDIO AMBIENTE & $\begin{array}{l}\text { Gestión de la Huella Medioambiental: } \\
\text { Scotiabank Ecoeficiente } \\
\text { Impulos de portafolio de productos: Línea de } \\
\text { Crédito Ambiental } \\
\text { Impulso de la asociación Ayudemos a } \\
\text { Reciclar, } \\
\text { Linea de Credito para cuidado de medio } \\
\text { ambiente }\end{array}$ & $\begin{array}{l}\text { Ejecuccion de un Inventario de Gas de efecto } \\
\text { invernadero (GEI), conocido como medición de } \\
\text { Huella de Carbono. Reciben asesoramiento de } \\
\text { la empresa Libélula especializada en gestión } \\
\text { de Cambio Climático }\end{array}$ & $\begin{array}{l}\text { Implementación de estaciones de reciclaje: } \\
\text { Primera empresa en implementarestaciones } \\
\text { de reciclaje para que clientes y } \\
\text { colaboradores depositen materiales } \\
\text { reciclables } \\
\text { Implementación del plan de eficiencia } \\
\text { energética } \\
\text { Consumo de agua, monitoreo y cumplimiento } \\
\text { con los valores máximos admisibles (VMA) }\end{array}$ \\
\hline $\begin{array}{l}\text { PRACTICAS JUSTAS DE } \\
\text { OPERACION }\end{array}$ & $\begin{array}{l}\text { Código de Conducta de Proveedores del } \\
\text { grupo Scotiabank, aprobado porla Gerencia } \\
\text { de responsabilidad social y porla Gerencia } \\
\text { de compras y contratos. } \\
\text { - Implementación del Manual de compras y } \\
\text { contratación de servicios,aprobado por la } \\
\text { Gerencia de compras y contratos } \\
\text { - Implementación del Manual de riesgo de } \\
\text { contratación outsourcing yacuerdos } \\
\text { similares }\end{array}$ & $\begin{array}{l}\text { RSE, alianza estratégica con proveedores para } \\
\text { construir relaciones comerciales competitivas } \\
\text { a largo plazo } \\
\text { - Política de Transparencia Comercial Sodimac } \\
\text { (TSC) con aliados comerciales: } \\
+ \text { Principios y ética comercial } \\
+ \text { Ámbito de aplicación } \\
+ \text { Soportes para aumentar y garantizar la } \\
\text { venta } \\
+ \text { Control de calidad de los productos y } \\
\text { servicios } \\
+ \text { Uso de marcas, patentes y similares } \\
+ \text { Normas de responsabilidad Social, } \\
\text { contraloría Transparencia Comercial } \\
\end{array}$ & 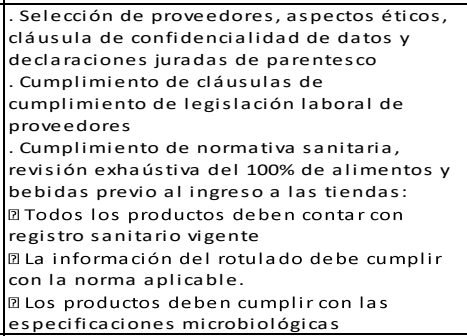 \\
\hline $\begin{array}{c}\text { ASUNTOS } \\
\text { CONSUMIDORES }\end{array}$ & $\begin{array}{l}\text { Ley de protección de datos personales } \\
\text { (LPDP) } \\
\text {. El cliente puede registrar los reclamos y } \\
\text { solicitudes a través de diferentes canales: } \\
\text { - Red de Agencias } \\
\text { - Banca telefónica } \\
\text { - Scotia en Línea } \\
\text { - Los reclamos se regsitran en el Sistema de } \\
\text { gestión de reclamos ylas solicitudes y } \\
\text { sugerencias son presentadas vía web. } \\
\text { En caso el cliente no quede satisfecho con } \\
\text { la respuesta a su reclamo, puede recurrira } \\
\text { una segunda instancia. }\end{array}$ & $\begin{array}{l}\text { Etica publicitaria y cumplimiento de normativa } \\
\text { legal } \\
\text {. Prácticas de comercio y marketing } \\
\text { responsable, enfocándose en el cliente como } \\
\text { el eje central del negocio, buscando garantizar } \\
\text { la satisfacción por el servicio y los productos } \\
\text { adquiridos } \\
\text { - Canales de escucha, a través de páginas web y } \\
\text { redes sociales, cartas de INDECOPI y medios de } \\
\text { prensa, encuestas online, ylibro de } \\
\text { Reclamaciones Web.. Política de protección y } \\
\text { privacidad de datos de clientes } \\
\text { - Control de calidad de productos, Sodimac Perú } \\
\text { realiza un control de calidad de productos } \\
\text { desde su ingreso al local hasta la venta y post } \\
\text { venta }\end{array}$ & $\begin{array}{l}\text { - Escucha de clientes, encuestas para evaluar } \\
\text { las recomendación de los clientes sobre las } \\
\text { tiendas ylas comparaciones con la } \\
\text { competencia. } \\
\text {. Canales de atención al cliente: Se cuenta } \\
\text { con un centro de atención en cada } \\
\text { supermercado. Se reciben reclamos y/o } \\
\text { sugerencias a través del call centerya tarvés } \\
\text { de medios digitales. } \\
\text { - Nivel de Recomendación, Se evaluan } \\
\text { permanentemente las recomendaciones de } \\
\text { los clientes } \\
\text { - participación en la Asociación Nacional de } \\
\text { Anunciantes del Perú (ANDA), se busca } \\
\text { practicaruna comunicación comercial } \\
\text { transparene yrespetuosa }\end{array}$ \\
\hline $\begin{array}{l}\text { PARTICIPACION ACTIVA Y } \\
\text { DESARROLLO DE LA } \\
\text { COMUNIDAD }\end{array}$ & $\begin{array}{l}\text { Enlace con la comunidad a través del } \\
\text { Programa comunitario de Scotiabank, } \\
\text { contribuye con el apoyo técnico y financiero } \\
\text { a iniciativas para que más personas } \\
\text { progresen en forma auto-sostenible } \\
\text { - Programa de inversion social } \\
\text { programa de voluntariado corporativo } \\
\text { - Campaña de recolección de fondos } \\
\text { Participación con auspicios Culturales } \\
\text { - Participación en modernizacion de } \\
\text { comisarias }\end{array}$ & $\begin{array}{l}\text { Metodología simple como modelo de } \\
\text { relacionamiento con la comunidad: } \\
\text { - Identificación de necesidades, recursos y } \\
\text { compromisos con la comunidad } \\
\text { - Elaboración de planes y diseño de estrategias, } \\
\text { programas y ycciones de relacionamiento } \\
\text { comunitario. } \\
\text { - Escucha y construcción de relaciones con la } \\
\text { comunidad para ejecutar programas yacciones } \\
\text { de interés común } \\
\text { - Evaluación y medición del impacto de las } \\
\text { iniciativas de gestión comunitaria. } \\
\text { - Identificación de áreas de oportunidad, } \\
\text { mejores prácticas e intercambio } \\
\text { - Apoyo de iniciativas relacionadas con la } \\
\text { salud, educación, desarrollo local, medio } \\
\text { ambiente yemprendimiento con la comunidad }\end{array}$ & $\begin{array}{l}\text { - Implementación del Programa bueno por } \\
\text { dentro, consiste en donaralimentos a } \\
\text { organizaciones sociales. En el } 2017 \text { se donó } \\
\text { más de } 530 \text { mil kg de comida. } \\
\text {. Programa recolección de tapitas, Perú } \\
\text { Champs } \\
\text { - Participación en la Teletón } \\
\text { "Magia porlos niños con cáncer", se apoya a } \\
\text { través del redondeo de vueltos, se reciben } \\
\text { las donaciones de los clientes. } \\
\text { - Colecta interna ALINEN } \\
\text { - Apoyo a la Fundación Peruana de Cáncerya } \\
\text { la Liga Peruana de Lucha contra el cáncer, } \\
\text { a poyo con donaciones de los clientespor el } \\
\text { redondeo de vueltos. } \\
\text {. Voluntariado SPSAUnido } \\
\text { - programa Una sola fuerza de voluntariado } \\
\text { para reconstruirlas casas de los empleados } \\
\text { afectados porlos desastres naturales. }\end{array}$ \\
\hline
\end{tabular}

Fuente: Informes oficiales de Great place to work y memorias de cada empresa 